\title{
Association of IL-6 -174 G>C Polymorphism with Susceptibility to Colorectal Cancer and Gastric Cancer: a Systematic Review and Meta-Analysis
}

\author{
Jamal Jafari-Nedooshan ${ }^{1}$, Seyed Alireza Dastgheib², Saeed Kargar ${ }^{1, *}$, Mohammad Zare ${ }^{1}$, \\ Ali Raee-Ezzabadi³, Naeimeh Heiranizadeh¹, Jalal Sadeghizadeh-Yazdi ${ }^{4}$, Hossein Neamatzadeh ${ }^{5,6}$
}

\begin{abstract}
Background: The $-174 \mathrm{G}>\mathrm{C}$ (rs1800795) polymorphism at interleukin 6 (IL-6) gene has been reported to be related with the occurrence of colorectal (CRC) and gastric (GC) cancers. However, the results had been conflicting and controversial. In order to give a comprehensive and precise result, we summarized available data to analyze the association of this polymorphism with CRC and GC risk.

Methods: A comprehensive literature search on PubMed, Elsevier Science Direct, and CNKI database was performed to identify all eligible studies up to May 15, 2019. The strength of association was assessed by odds ratios (ORs) with $95 \%$ confidence intervals (CI).

Results: A total of 29 case-control studies including 16 studies with 7,560 cases and 9,574 controls on CRC and 13 studies with 1,445 cases and 2,918 controls on GC were selected. Overall, pooled data showed that the IL- $6-174$ G $>C$ polymorphism was not significantly associated with increased risk of CRC and GC in overall. When stratified by ethnicity, we found a statistically significant association between the IL-6 -174 G>C polymorphism and CRC risk in Asians (CC vs. GG: OR = 1.860, 95\% Cl 1.061-3.258, $\mathrm{p}=0.030$; and CC vs. CG+GG: OR = 1.941, $95 \% \mathrm{Cl} 1.131-3.331, \mathrm{p}=0.016)$.

Conclusion: The meta-analysis suggests that IL-6 -174G $>C$ polymorphism was not significantly associated with the increased risk of CRC and GC in overall population. However, the results showed that IL- $6-174 \mathrm{G}>\mathrm{C}$ polymorphism may be associated with risk of GC in Asians. Further studies including a larger sample size will be necessary to clarify these results.
\end{abstract}

\section{KEYWORDS}

colorectal cancer; gastric cancer; interleukin 6; association; meta-analysis

AUTHOR AFFILIATIONS

${ }^{1}$ Department of General Surgery, Shahid Sadoughi University of Medical Sciences, Yazd, Iran

2 Department of Medical Genetics, School of Medicine, Shiraz Sadoughi University of Medical Sciences, Shiraz, Iran

${ }^{3}$ Department of Emergency Medicine, Shahid Sadoughi University of Medical Sciences, Yazd, Iran

${ }^{4}$ Department of Food Science and Technology, School of Public Health, Shahid Sadoughi University of Medical Sciences, Yazd, Iran

${ }^{5}$ Mother and Newborn Health Research Center, Shahid Sadoughi University of Medical Sciences, Yazd, Iran

${ }^{6}$ Department of Medical Genetics, Shahid Sadoughi University of Medical Sciences, Yazd, Iran

* Corresponding author: Department of General Surgery, Shahid Sadoughi University of Medical Sciences, Yazd, Iran; e-mail: saeedkargar@yahoo.com

Received: 2 July 2019

Accepted: 4 September 2019

Published online: 10 February 2020

Acta Medica (Hradec Králové) 2019; 62(4): 137-146

https://doi.org/10.14712/18059694.2020.2

(c) 2019 The Authors. This is an open-access article distributed under the terms of the Creative Commons Attribution License (http://creativecommons.org/licenses/by/4.0), which permits unrestricted use, distribution, and reproduction in any medium, provided the original author and source are credited. 


\section{INTRODUCTION}

Digestive system cancers especially colorectal cancer (CRC) and Gastric cancer (GC) are the most common causes of cancer-related death worldwide (1-3). CRC and GC were the fourth and second most common causes of cancer-related mortality worldwide in 2016 , respectively (4, 5). The exact mechanism of CRC and GC is still not fully understood. However, CRC and GC are multifactorial and multistep diseases caused by complex interactions between environmental triggers and genetic factors $(6,7)$. To date, a wide range of gastrointestinal cancer susceptibility gene variations have been evaluated. Interleukin 6 (IL-6) gene promoter region polymorphisms have already been correlated to increased risks of developing CRC and GC $(8,9)$.

IL-6 is a pleiotropic cytokine with a wide range of biological activities in immune regulation, hematopoiesis, inflammation and oncogenesis. IL-6 is implicated in a wide variety of inflammation-associated disease states, such as diabetes mellitus, systemic juvenile rheumatoid arthritis and malignant diseases. The human IL- 6 gene is mapped to chromosome 7p21-24, with an upstream promoter containing $303 \mathrm{bp}$, contains five exons and spans approximately $6.2 \mathrm{~kb}$ (10). Accumulating evidence indicates pathological roles for IL-6 in different malignancies, such as breast, vulvar, ovarian, hepatocellular, lung, gastric and colorectal cancer (11).

To date, several polymorphisms in the promoter region of the IL-6 gene including $-598 \mathrm{~A}>\mathrm{G},-597 \mathrm{G}>\mathrm{A},-572 \mathrm{C}>\mathrm{G}$, and $-174 \mathrm{G}>\mathrm{C}$ have been identified and are implicated in the increased level of IL-6. Of these polymorphisms, -174 $\mathrm{G}>\mathrm{C}$ (rs1800795) is the most studied functional polymorphism in different malignancies. IL-6 -174 G>C is demonstrated to impact the adherence of the glucocorticoid receptor and then results in repressive transcriptional activation. Lots of studies have reported the role of IL-6 -174 polymorphism in the predisposition to CRC and GC. However, these studies results are inconclusive and also inconsistent. This may be because of inadequate sample sizes, patient selection, genotyping methods, and ethnicity of the populations studied. Moreover, an individual study may be insufficient to evaluate the potential small effect of the IL-6 -174 G>C polymorphism on risk of CRC and GC. Therefore, we performed a meta-analysis of all available eligible case-control studies to verify the precise association of the IL-6 -174 G>C (rs1800795) polymorphism with $\mathrm{CRC}$ and GC risk.

\section{MATERIAL AND METHODS}

\section{LITERATURE COLLECTION AND SCREENING}

A flow-diagram outlining the identification, screening, eligibility, and final datasets was constructed according to Preferred Reporting Items for Systematic Reviews and Meta-analyses (PRISMA) 2009 guidelines. To identify all articles that evaluated the association of IL-6 -174 $\mathrm{G}>\mathrm{C}$ polymorphism with CRC and GC risk, we performed a comprehensive literature search of the PubMed, EMBASE, Elsevier Science Direct, Google scholar, Chinese Bi- omedical Literature database, China National Knowledge Infrastructure database (CNKI), and Wanfang database up to May 15, 2019. The following keywords and terms were used: ("colorectal cancer" Or "CRC" OR "bowel cancer" OR "colon cancer") AND ("gastric cancer" OR "GC" OR "stomach cancer") AND (Interleukin 6 OR IL-6 OR “-174G>C" OR "rs1800795") AND ("gene" OR "polymorphism" OR "mutation" OR "variation"). In addition, reference list of obtained literatures was reviewed to ensure that no relevant studies were missed.

\section{INCLUSION AND EXCLUSION CRITERIA}

The inclusion criteria for the present study were as follows: 1) published case-control or cohort studies; 2) studies evaluated the association of IL-6 -174 G>C polymorphism with CRC and GC; 3 ) studies with sufficient data to calculate the odds ratio (OR) and 95\% confidence interval (CI). Accordingly, the following exclusion criteria were used: 1) abstracts, posters, case reports, reviews, and letter to editors; 2) case only studies, sibling or linkage studies; 3 ) the study reported duplicated data or containing overlapping data.

\section{DATA EXTRACTION}

The data from the published studies were extracted independently by two of the authors, and the disagreement was resolved by a discussion involving a senior author. For each study, the following data were collected: first author's name, year of publication, country, ethnicity (Caucasian, Asian, African and others), sources of healthy controls, number of cases and controls, genotyping methods, allele numbers and genotype distributions in cases and controls, minor allele frequencies (MAFs) in control subjects, and the results of Hardy-Weinberg equilibrium (HWE) test.

\section{STATISTICAL ANALYSIS}

An ethical approval was not necessary as this study was a meta-analysis based on previous studies. The association of IL-6 -174 G>C polymorphism with CRC and GC risk was measured by ORs and its corresponding $95 \%$ CIs. The estimates of pooled ORs were obtained by calculating a weighted average of OR from each study and the significance of pooled ORs was determined by the Z-test. In this meta-analysis, the pooled ORs for IL- $6-174 \mathrm{G}>\mathrm{C}$ polymorphism was calculated under five genetic models, i.e., allele (C vs. $G$ ), homozygote (CC vs. GG), heterozygote (CG vs. $G G)$, dominant (CC+CG vs. GG) and recessive (CC vs. $C G+G G$ ). Between-studies heterogeneity was assessed by a Chi-squared Q-test and $\mathrm{I}^{2}$ statistics $(\mathrm{P}<0.05)$. Additionally, the $\mathrm{I}^{2}$-value was applied to quantitatively evaluate the heterogeneity $\left(\mathrm{I}^{2}<25 \%\right.$, low heterogeneity; $25 \% \leq \mathrm{I}^{2} \leq 75 \%$, moderate heterogeneity; $\mathrm{I}^{2}>75 \%$, high heterogeneity). The fixed-effects model (the Mantel-Haenszel method) and the random-effects model (the DerSimonian-Laird method) were utilized to pool ORs. Sensitivity analysis was used by omitting individual studies each time to assess the stability of the pooled results. The Hardy-Weinberg equi- 
librium (HWE) test for each study was performed using chi-square test), and $\mathrm{P}<0.05$ was considered to indicate significant disequilibrium. We carried out subgroup analysis by cancer type, ethnicity, genotyping methods, source of controls and HWE (fall in HWE). Begg's funnel plot and Egger's test were used to evaluate the publication bias in the meta-analysis, in which $\mathrm{P}<0.05$ indicated that the result was statistically significant. All statistical analyses were performed using the comprehensive meta-analysis (CMA) software (version 2.0, Biostat, USA). Two-sided $\mathrm{P}<0.05$ was considered statistically significant.

\section{RESULTS}

\section{LITERATURE SELECTION}

AND STUDY CHARACTERISTICS

A total of 118 articles were identified through the initial search in the database and by hand searching. As shown in Figure 1, after carefully screening the title and abstracts of the initial publications, 20 studies were promptly excluded. Consequently, 29 case-control studies were included in this meta-analysis. The characteristics of each study are summarized in Table 1. Of those studies, 16 studies with

Tab. 1 Main characteristics of studies included in this meta-analysis.

\begin{tabular}{|c|c|c|c|c|c|c|c|c|c|c|c|c|c|c|c|c|}
\hline \multirow{3}{*}{$\begin{array}{l}\text { First Author } \\
\begin{array}{l}\text { Colorectal } \\
\text { Cancer }\end{array}\end{array}$} & \multirow{3}{*}{$\begin{array}{l}\text { Country } \\
\text { (Ethnicity) }\end{array}$} & \multirow{3}{*}{$\begin{array}{l}\text { Genotyping } \\
\text { Method }\end{array}$} & \multirow{3}{*}{ SOC } & \multirow{3}{*}{$\begin{array}{l}\text { Case/ } \\
\text { Control }\end{array}$} & \multicolumn{5}{|c|}{ Cases } & \multicolumn{5}{|c|}{ Controls } & \multirow{3}{*}{ MAFs } & \multirow{3}{*}{ HWE } \\
\hline & & & & & \multicolumn{3}{|c|}{ Genotypes } & \multicolumn{2}{|c|}{ Allele } & \multicolumn{3}{|c|}{ Genotypes } & \multicolumn{2}{|c|}{ Allele } & & \\
\hline & & & & & GG & GC & CC & G & C & GG & GC & $\mathrm{CC}$ & G & C & & \\
\hline Landi 2003 (12) & $\begin{array}{l}\text { Spain } \\
\text { (Caucasian) }\end{array}$ & TaqMan & PB & $361 / 311$ & 133 & 180 & 48 & 446 & 276 & 145 & 133 & 33 & 423 & 199 & 0.319 & 0.761 \\
\hline $\begin{array}{l}\text { Theodoropoulos } \\
2006(13)\end{array}$ & $\begin{array}{l}\text { Greece } \\
\text { (Caucasian) }\end{array}$ & PCR-RFLP & NS & $222 / 200$ & 111 & 76 & 35 & 298 & 146 & 64 & 86 & 50 & 214 & 186 & 0.465 & 0.054 \\
\hline $\begin{array}{l}\text { Gunter } 2006 \\
(14)\end{array}$ & $\begin{array}{l}\text { USA } \\
\text { (Caucasian) }\end{array}$ & TaqMan & $\mathrm{HB}$ & 204/190 & 79 & 90 & 35 & 248 & 160 & 83 & 81 & 26 & 247 & 133 & 0.350 & 0.384 \\
\hline $\begin{array}{l}\text { Gaustadnes } \\
2006(15)\end{array}$ & \begin{tabular}{|l|} 
Denmark \\
(Caucasian)
\end{tabular} & CE & PB & $230 / 540$ & 64 & 115 & 51 & 243 & 217 & 184 & 263 & 93 & 631 & 449 & 0.415 & 0.952 \\
\hline $\begin{array}{l}\text { Slattery } 2007 \\
(16)\end{array}$ & $\begin{array}{l}\text { USA } \\
\text { (Caucasian) }\end{array}$ & TaqMan & $\mathrm{HB}$ & 777/995 & 321 & 347 & 109 & 989 & 565 & 411 & 438 & 146 & 1260 & 730 & 0.366 & 0.098 \\
\hline Vogel 2007 (17) & $\begin{array}{l}\text { Denmark } \\
\text { (Caucasian) }\end{array}$ & Probe & $\mathrm{HB}$ & $355 / 753$ & 98 & 168 & 89 & 364 & 346 & 204 & 364 & 185 & 772 & 734 & 0.487 & 0.371 \\
\hline $\begin{array}{l}\text { Wilkening } 2008 \\
(18)\end{array}$ & $\begin{array}{l}\text { Sweden } \\
\text { (Caucasian) }\end{array}$ & TaqMan & $\mathrm{HB}$ & $303 / 580$ & 79 & 163 & 61 & 321 & 285 & 162 & 297 & 121 & 621 & 539 & 0.464 & 0.480 \\
\hline Kury 2008 (19) & \begin{tabular}{|l|} 
France \\
(Caucasian)
\end{tabular} & TaqMan & PB & $1023 / 1121$ & 363 & 489 & 171 & 1215 & 831 & 435 & 504 & 182 & 1374 & 868 & 0.387 & 0.078 \\
\hline $\begin{array}{l}\text { Slattery } 2009 \\
(20)\end{array}$ & $\begin{array}{l}\text { USA } \\
\text { (Caucasian) }\end{array}$ & TaqMan & $\mathrm{HB}$ & $1573 / 1972$ & 631 & 696 & 246 & 1958 & 1188 & 728 & 897 & 347 & 2353 & 1591 & 0.403 & 0.014 \\
\hline $\begin{array}{l}\text { Tsilidis } 2009 \\
(21)\end{array}$ & $\begin{array}{l}\text { USA } \\
\text { (Caucasian) }\end{array}$ & TaqMan & $\mathrm{HB}$ & $200 / 354$ & 68 & 93 & 39 & 229 & 171 & 113 & 170 & 71 & 396 & 312 & 0.440 & 0.626 \\
\hline Vasku 2009 (22) & $\begin{array}{l}\text { Czech } \\
\text { (Caucasian) }\end{array}$ & PCR-RFLP & $\mathrm{HB}$ & $100 / 100$ & 32 & 46 & 22 & 110 & 90 & 31 & 47 & 22 & 109 & 91 & 0.455 & 0.600 \\
\hline $\begin{array}{l}\text { Ognjanovic } \\
2010(23)\end{array}$ & $\begin{array}{l}\text { USA } \\
\text { (Caucasian) } \\
\end{array}$ & TaqMan & PB & $117 / 221$ & 71 & 46 & 0 & 188 & 46 & 103 & 118 & 0 & 324 & 118 & 0.267 & $\leq 0.001$ \\
\hline Cacev 2010 (24) & $\begin{array}{l}\text { Croatia } \\
\text { (Caucasian) }\end{array}$ & PCR-RFLP & $\mathrm{HB}$ & $160 / 160$ & 64 & 70 & 26 & 198 & 122 & 68 & 75 & 17 & 211 & 109 & 0.340 & 0.581 \\
\hline Abuli 2010 (25) & $\begin{array}{l}\text { Spain } \\
\text { (Caucasian) }\end{array}$ & TaqMan & $\mathrm{HB}$ & $1405 / 1388$ & 586 & 635 & 184 & 1807 & 1003 & 593 & 623 & 172 & 1809 & 967 & 0.348 & 0.672 \\
\hline $\begin{array}{l}\text { Basavaraju } 2015 \\
(26)\end{array}$ & $\begin{array}{l}\text { Scotland } \\
\text { (Caucasian) }\end{array}$ & TaqMan & $\mathrm{HB}$ & $388 / 495$ & 140 & 184 & 64 & 464 & 312 & 172 & 245 & 78 & 589 & 401 & 0.405 & 0.549 \\
\hline $\begin{array}{l}\text { Banday } 2017 \\
(27)\end{array}$ & $\begin{array}{l}\text { Kashmiri } \\
\text { (Asian) }\end{array}$ & PCR-RFLP & PHB & $142 / 194$ & 85 & 43 & 14 & 213 & 71 & 145 & 46 & 3 & 316 & 52 & 0.134 & 0.764 \\
\hline \multicolumn{17}{|l|}{ Gastric Cancer } \\
\hline $\begin{array}{l}\text { El-Omar } 2003 \\
(28)\end{array}$ & $\begin{array}{l}\text { USA } \\
\text { (Caucasian) } \\
\end{array}$ & TaqMan & PB & $213 / 209$ & 88 & 91 & 43 & 267 & 177 & 83 & 98 & 28 & 264 & 154 & 0.368 & 0.912 \\
\hline $\begin{array}{l}\text { Hwang } 2003 \\
(29)\end{array}$ & $\begin{array}{l}\text { USA } \\
\text { (Caucasian) } \\
\end{array}$ & PCR-RFLP & $\mathrm{HB}$ & $30 / 30$ & 19 & 9 & 2 & 37 & 13 & 22 & 8 & 0 & 52 & 8 & 0.133 & 0.399 \\
\hline Hwang 2003 & $\begin{array}{l}\text { USA } \\
\text { (Asian) }\end{array}$ & PCR-RFLP & $\mathrm{HB}$ & $30 / 30$ & 30 & 0 & 0 & 60 & 0 & 30 & 0 & 0 & 60 & 0 & 0.000 & NA \\
\hline $\begin{array}{l}\text { Kamangar } 2006 \\
(30)\end{array}$ & \begin{tabular}{|l|} 
Finland \\
(Caucasian) \\
\end{tabular} & TaqMan & PB & $102 / 152$ & 21 & 54 & 27 & 96 & 108 & 51 & 58 & 43 & 160 & 144 & 0.473 & 0.003 \\
\hline Xing 2006 (31) & $\begin{array}{l}\text { China } \\
\text { (Asian) }\end{array}$ & PCR-RFLP & PB & $65 / 71$ & 62 & 3 & 0 & 127 & 3 & 67 & 4 & 0 & 138 & 4 & 0.028 & 0.807 \\
\hline
\end{tabular}




\begin{tabular}{|l|l|l|l|l|l|l|l|l|l|l|l|l|l|l|l|l|}
\hline Deans 2007 (32) & $\begin{array}{l}\text { UK } \\
\text { (Caucasian) }\end{array}$ & TaqMan & HB & $197 / 224$ & 71 & 83 & 43 & 225 & 169 & 79 & 101 & 44 & 259 & 189 & 0.421 & 0.257 \\
\hline Gatti 2007 (33) & $\begin{array}{l}\text { Brazil } \\
\text { (Mixed) }\end{array}$ & PCR-RFLP & HB & $56 / 112$ & 42 & 13 & 1 & 97 & 15 & 48 & 53 & 11 & 73 & 39 & 0.334 & 0.509 \\
\hline $\begin{array}{l}\text { Crusius } 2008 \\
(34)\end{array}$ & $\begin{array}{l}\text { France } \\
\text { (Caucasian) }\end{array}$ & PCR-RFLP & PB & $243 / 1138$ & 78 & 122 & 43 & 278 & 208 & 415 & 517 & 206 & 1347 & 929 & 0.408 & 0.044 \\
\hline Zhao 2010 (35) & $\begin{array}{l}\text { China } \\
\text { (Asian) }\end{array}$ & PCR-RFLP & NS & $142 / 200$ & 105 & 37 & 0 & 247 & 37 & 198 & 2 & 0 & 398 & 2 & 0.005 & 0.943 \\
\hline $\begin{array}{l}\text { Pohjanen 2013 } \\
\text { (36) }\end{array}$ & $\begin{array}{l}\text { Finland } \\
\text { (Caucasian) }\end{array}$ & PCR-RFLP & PB & $56 / 179$ & 14 & 34 & 8 & 62 & 50 & 37 & 86 & 56 & 160 & 198 & 0.553 & 0.706 \\
\hline Cao 2014 (37) & $\begin{array}{l}\text { China } \\
\text { (Asian) }\end{array}$ & NS & NS & $162 / 162$ & 72 & 62 & 28 & 206 & 188 & 87 & 59 & 16 & 233 & 91 & 0.280 & 0.210 \\
\hline $\begin{array}{l}\text { Sampaio 2015 } \\
\text { (38) }\end{array}$ & $\begin{array}{l}\text { Portugal } \\
\text { (Caucasian) }\end{array}$ & SSP-PCR & NS & $50 / 50$ & 17 & 25 & 8 & 59 & 41 & 19 & 25 & 6 & 63 & 37 & 0.370 & 0.608 \\
\hline Attar 2017 (39) & Iran (Asian) & SSP-PCR & HB & $100 / 361$ & 60 & 30 & 7 & 150 & 47 & 161 & 187 & 13 & 509 & 213 & 0.295 & $\leq 0.001$ \\
\hline
\end{tabular}

PCR: Polymerase Chain Reaction Restriction; PCR-RFLP: Polymerase Chain Reaction Restriction Fragment Length Polymorphism; CE: Primer extension and capillary electrophoresis; HB: Hospital Based; PB: Population Based; NS: Not stated; MAFs: Minor Allele Frequencies; HWE: Hardy-Weinberg Equilibrium; NA: Not Applicable.
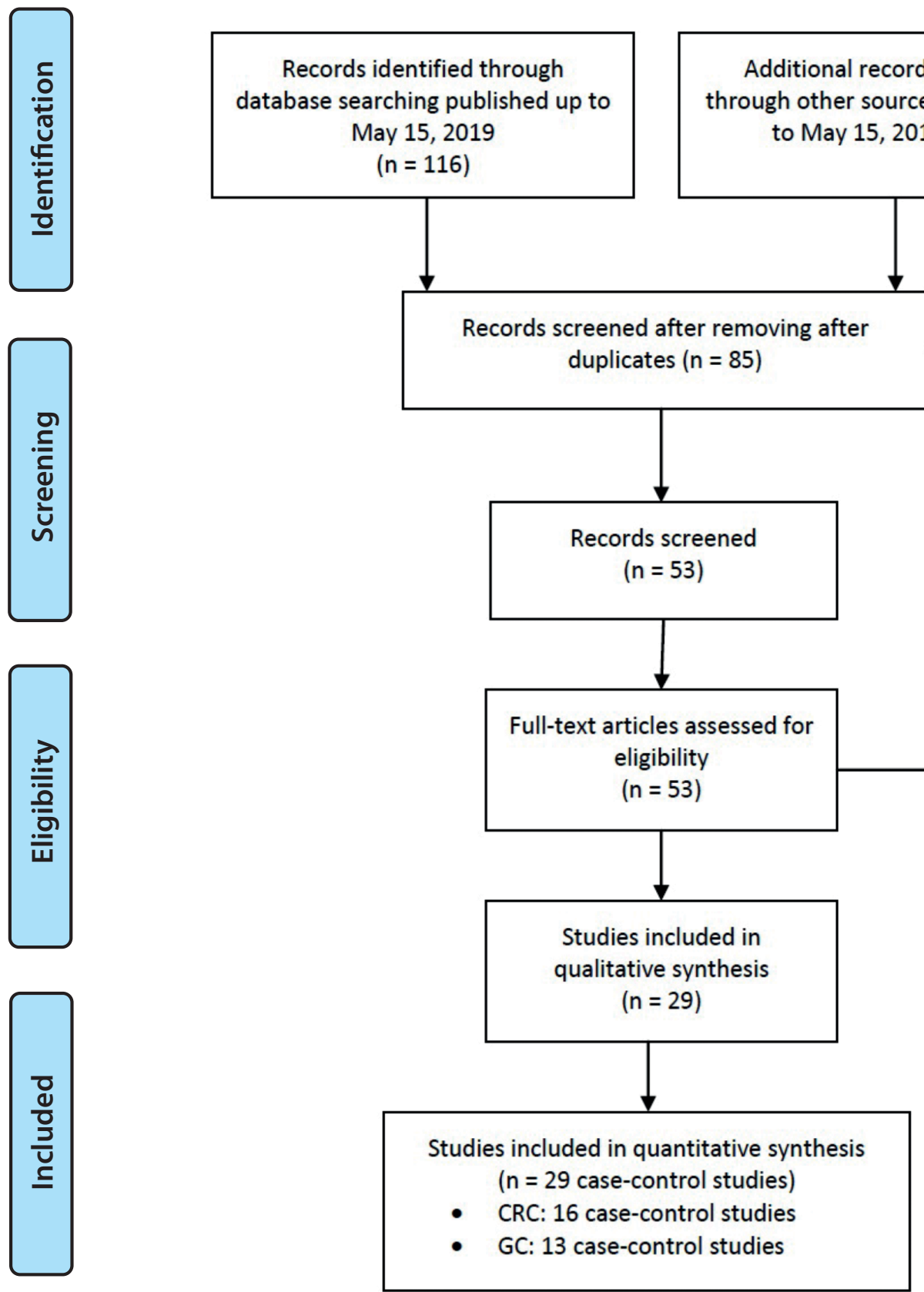

Full-text articles excluded, with reasons $(n=24)$ Review, case reports, letters to editors, evaluated other disease instead of CRC and GC, other SNPs of IL-6 gene.

Fig. 1 The study selection and inclusion process. 
7,560 cases and 9,574 controls were on CRC (12-27), and 13 studies with 1,445 cases and 2,918 controls were on GC (28-39). These included studies were published between 2003 and 2017. Twenty-two studies populations come from Caucasians, six studies were Asians, and only one from mixed populations. The genotype and allele distributions of the IL-6 -174 G>C polymorphism were shown in Table 1. Genotype distributions in the controls of all studies were in HWE except for five studies (Table 1).

\section{QUANTITATIVE SYNTHESIS}

IL-6 -174G>C Polymorphism and CRC

Table 2 shows the results of the association between IL-6 -174 G>C polymorphism and CRC risk. Overall, the pooled data showed that the IL-6 -174 G>C polymorphism was not significantly associated with an increased risk of CRC under all five genetic models, i.e., allele (C vs. G: OR = $1.028,95 \%$ CI 0.936-1.128, $\mathrm{p}=0.566$ ), homozygote (CC vs.
GG: OR $=1.068,95 \%$ CI 0.902-1.263, $\mathrm{p}=0.447)$, heterozygote (CG vs. GG: OR = 1.007, 95\% CI 0.902-1.124, p = 0.904), dominant (CC+CG vs. GG: OR = 1.021, 95\% CI 0.903-1.155, $\mathrm{p}=0.737$, Figure $2 \mathrm{~A}$ ), and recessive (CC vs. CG+GG: OR = $1.037,95 \%$ CI 0.917-1.171, p = 0.564). When further analyzed by genotyping methods, we have not found a significant association between IL-6 -174 G>C polymorphism and CRC. However, subgroup analysis by source of controls showed a significant association between IL-6 -174 G>C polymorphism and risk of CRC under the homozygote model (CC vs. $G G$ : OR $=1.273,95 \%$ CI 1.042-1.556, $\mathrm{p}=0.018$ ) in population-based $(\mathrm{PB})$ group studies.

\section{IL-6 -174G>C Polymorphism and GC}

Table 3 shows the results of the association between IL-6 -174 G>C polymorphism and GC risk. Overall, the pooled data showed that there was no significant association between IL-6 -174 G>C polymorphism and increased risk of GC under all five genetic models, i.e., allele (C vs. G:

Tab. 2 Meta-analysis results of association of IL-6 -174 G>C polymorphism with CRC risk.

\begin{tabular}{|c|c|c|c|c|c|c|c|c|c|c|}
\hline \multirow{2}{*}{ Subgroup } & \multirow{2}{*}{$\begin{array}{l}\text { Genetic } \\
\text { Model }\end{array}$} & \multirow{2}{*}{$\begin{array}{l}\text { Type of } \\
\text { Model }\end{array}$} & \multicolumn{2}{|c|}{ Heterogeneity } & \multicolumn{4}{|c|}{ Odds Ratio } & \multicolumn{2}{|c|}{ Publication Bias } \\
\hline & & & $\mathrm{I}^{2}(\%)$ & $\mathbf{P}_{\mathrm{H}}$ & OR & $95 \% \mathrm{Cl}$ & $Z_{\text {test }}$ & $P_{\text {OR }}$ & $P_{\text {Beggs }}$ & $P_{\text {Eggers }}$ \\
\hline \multirow[t]{5}{*}{ Overall } & C vs. G & Random & 72.34 & $\leq 0.001$ & 1.028 & $0.936-1.128$ & 0.574 & 0.566 & 0.558 & 0.491 \\
\hline & CC vs. GG & Random & 62.56 & 0.001 & 1.068 & $0.902-1.263$ & 0.760 & 0.447 & 0.198 & 0.110 \\
\hline & CG vs. GG & Random & 53.39 & 0.006 & 1.007 & $0.902-1.124$ & 0.121 & 0.904 & 1.000 & 0.916 \\
\hline & $\mathrm{CC}+\mathrm{CG}$ vs. GG & Random & 67.49 & $\leq 0.001$ & 1.021 & $0.903-1.155$ & 0.336 & 0.737 & 0.964 & 0.781 \\
\hline & $\mathrm{CC}$ vs. $\mathrm{CG}+\mathrm{GG}$ & Random & 42.66 & 0.041 & 1.037 & $0.917-1.171$ & 0.576 & 0.564 & 0.113 & 0.044 \\
\hline \multicolumn{11}{|c|}{ Genotyping Method } \\
\hline \multirow[t]{5}{*}{ TaqMan } & C vs. G & Random & 51.33 & 0.030 & 1.012 & 0.937-1.093 & 0.299 & 0.765 & 1.000 & 0.696 \\
\hline & CC vs. GG & Fixed & 21.34 & 0.253 & 1.002 & $0.904-1.110$ & 0.031 & 0.975 & 0.251 & 0.095 \\
\hline & CG vs. GG & Random & 49.53 & 0.037 & 1.017 & $0.908-1.139$ & 0.295 & 0.768 & 1.000 & 0.969 \\
\hline & $\mathrm{CC}+\mathrm{CG}$ vs. GG & Random & 56.96 & 0.013 & 1.018 & $0.907-1.143$ & v0.303 & 0.762 & 0.858 & 0.840 \\
\hline & CC vs. CG+GG & Fixed & 0.00 & 0.746 & 0.988 & $0.900-1.086$ & -0.243 & 0.808 & 0.175 & 0.055 \\
\hline \multirow[t]{5}{*}{ PCR-RFLP } & C vs. G & Random & 90.52 & $\leq 0.001$ & 1.081 & $0.619-1.886$ & 0.273 & 0.785 & 0.308 & 0.196 \\
\hline & CC vs. GG & Random & 86.82 & $\leq 0.001$ & 1.345 & $0.472-3.829$ & 0.555 & 0.579 & 0.308 & 0.072 \\
\hline & CG vs. GG & Random & 74.52 & 0.008 & 0.919 & $0.558-1.512$ & -0.333 & 0.739 & 0.734 & 0.603 \\
\hline & $\mathrm{CC}+\mathrm{CG}$ vs. GG & Random & 86.40 & $\leq 0.001$ & 0.991 & $0.525-1.871$ & -0.028 & 0.978 & 0.734 & 0.565 \\
\hline & $C C$ vs. $C G+G G$ & Random & 81.96 & 0.001 & 1.374 & $0.602-3.137$ & 0.754 & 0.451 & 0.308 & 0.055 \\
\hline \multicolumn{11}{|c|}{ Source of Controls } \\
\hline \multirow[t]{5}{*}{ HB } & C vs. G & Fixed & 0.00 & 0.548 & 0.983 & $0.934-1.036$ & -0.636 & 0.525 & 0.474 & 0.102 \\
\hline & CC vs. GG & Fixed & 0.00 & 0.572 & 0.971 & $0.872-1.080$ & -0.548 & 0.584 & 0.210 & 0.053 \\
\hline & CG vs. GG & Fixed & 0.00 & 0.934 & 0.975 & $0.902-1.055$ & -0.621 & 0.534 & 0.591 & 0.503 \\
\hline & $\mathrm{CC}+\mathrm{CG}$ vs. GG & Fixed & 0.00 & 0.774 & 0.975 & $0.906-1.050$ & -0.668 & 0.504 & 0.720 & 0.241 \\
\hline & CC vs. CG+GG & Fixed & 0.00 & 0.738 & 0.984 & $0.893-1.085$ & -0.320 & 0.749 & 0.107 & 0.032 \\
\hline \multirow[t]{5}{*}{ PB } & C vs. G & Random & 70.12 & 0.018 & 1.097 & $0.899-1.338$ & 0.911 & 0.362 & 1.000 & 0.721 \\
\hline & CC vs. GG & Fixed & 21.43 & 0.280 & 1.273 & $1.042-1.556$ & 2.364 & 0.018 & 1.000 & 0.096 \\
\hline & CG vs. GG & Random & 74.65 & 0.008 & 1.089 & $0.796-1.490$ & 0.532 & 0.595 & 0.734 & 0.602 \\
\hline & $\mathrm{CC}+\mathrm{CG}$ vs. $\mathrm{GG}$ & Random & 76.71 & 0.005 & 1.111 & $0.812-1.519$ & 0.657 & 0.511 & 0.734 & 0.666 \\
\hline & CC vs. CG+GG & Fixed & 0.00 & 0.400 & 1.139 & $0.950-1.365$ & 1.407 & 0.159 & 1.000 & 0.267 \\
\hline
\end{tabular}

CRC: colorectal cancer; PCR-RFLP: Polymerase Chain Reaction Restriction Fragment Length Polymorphism; HB: Hospital Based; PB: Population Based. 
OR $=1.282,95 \%$ CI 0.927-1.774, $\mathrm{p}=0.134$ ), homozygote (CC vs. GG: OR $=1.209,95 \%$ CI 0.967-1.512, $\mathrm{p}=0.096)$, heterozygote (CG vs. GG: OR = 1.097, 95\% CI 0.736-1.634, $\mathrm{p}=0.649$ ), dominant (CC+CG vs. GG: OR = 1.117, 95\% CI 0.759-1.644, $\mathrm{p}=0.573$ ), and recessive (CC vs. $C G+G G: O R=1.115,95 \%$ CI $0.803-1.548, p=0.517$, Figure $2 B$ ). When further analyz- ed by ethnicity, we found a statistically significant association between IL-6 -174 G>C polymorphism and CRC risk under two genetic models i.e., homozygote (CC vs. GG: OR $=1.860,95 \%$ CI $1.061-3.258, \mathrm{p}=0.030)$ and recessive (CC vs. CG+GG: OR = 1.941, 95\% CI 1.131-3.331, $\mathrm{p}=0.016)$ in Asians, but not in Caucasians.

A

\begin{tabular}{lrrrrrr} 
Study name & \multicolumn{5}{c}{ Statistics for each study } \\
\cline { 1 - 6 } & $\begin{array}{c}\text { Odds } \\
\text { ratio }\end{array}$ & $\begin{array}{c}\text { Lower } \\
\text { limit }\end{array}$ & $\begin{array}{c}\text { Upper } \\
\text { limit }\end{array}$ & Z-Value & p-Value \\
Landi 2003 & 1.497 & 1.100 & 2.039 & 2.562 & 0.010 \\
Theodoropoulos 2006 & 0.471 & 0.316 & 0.700 & $3.723-$ & 0.000 \\
Gunter 2006 & 1.227 & 0.821 & 1.835 & 0.999 & 0.318 \\
Gaustadnes 2006 & 1.341 & 0.955 & 1.881 & 1.695 & 0.090 \\
Slattery 2007 & 1.000 & 0.826 & 1.210 & -0.003 & 0.998 \\
Vogel 2007 & 0.974 & 0.734 & 1.293 & $0.179-$ & 0.858 \\
Wilkening 2008 & 1.099 & 0.803 & 1.504 & 0.588 & 0.556 \\
Kury 2008 & 1.153 & 0.967 & 1.374 & 1.588 & 0.112 \\
Slattery 2009 & 0.874 & 0.762 & 1.001 & $1.945-$ & 0.052 \\
Tsilidis 2009 & 0.910 & 0.630 & 1.315 & $0.501-$ & 0.616 \\
Vasku 2009 & 0.955 & 0.526 & 1.734 & $0.152-$ & 0.879 \\
Ognjanovic 2010 & 0.566 & 0.359 & 0.892 & $2.453-$ & 0.014 \\
Cacev 2010 & 1.109 & 0.710 & 1.731 & 0.454 & 0.650 \\
Abuli 2010 & 1.042 & 0.897 & 1.211 & 0.543 & 0.587 \\
Basavaraju 2015 & 0.943 & 0.715 & 1.245 & $0.412-$ & 0.680 \\
Banday 2017 & 1.984 & 1.245 & 3.163 & 2.880 & 0.004 \\
& 1.021 & 0.903 & 1.155 & 0.336 & 0.737
\end{tabular}

Fig. 2 Forest plot for association of IL-6 -174 G>C polymorphism with CRC and GC risk in random-effects model. A: CRC (dominant model: CC+CG vs. GG); B: GC (recessive model: CC vs. CG+GG).

Tab. 3 Meta-analysis results of association of IL-6 -174 G>C polymorphism with GC risk.

\begin{tabular}{|c|c|c|c|c|c|c|c|c|c|c|}
\hline \multirow{2}{*}{ Subgroup } & \multirow{2}{*}{$\begin{array}{l}\text { Genetic } \\
\text { Model }\end{array}$} & \multirow{2}{*}{$\begin{array}{l}\text { Type of } \\
\text { Model }\end{array}$} & \multicolumn{2}{|c|}{ Heterogeneity } & \multicolumn{4}{|c|}{ Odds Ratio } & \multicolumn{2}{|c|}{ Publication Bias } \\
\hline & & & $I^{2}(\%)$ & $\mathbf{P}_{\mathrm{H}}$ & OR & $95 \% \mathrm{Cl}$ & $Z_{\text {test }}$ & $P_{\text {OR }}$ & $\mathbf{P}_{\text {Beggs }}$ & $P_{\text {Eggers }}$ \\
\hline \multirow[t]{5}{*}{ Overall } & C vs. G & Random & 86.50 & $\leq 0.001$ & 1.282 & $0.927-1.774$ & 1.499 & 0.134 & 0.731 & 0.561 \\
\hline & CC vs. GG & Random & 43.05 & 0.071 & 1.209 & $0.967-1.512$ & 1.666 & 0.096 & 0.858 & 0.646 \\
\hline & CG vs. GG & Random & 80.63 & $\leq 0.001$ & 1.097 & $0.736-1.634$ & 0.456 & 0.649 & 0.631 & 0.527 \\
\hline & $\mathrm{CC}+\mathrm{CG}$ vs. GG & Random & 81.53 & $\leq 0.001$ & 1.117 & $0.759-1.644$ & 0.563 & 0.573 & 1.000 & 0.653 \\
\hline & CC vs. $C G+G G$ & Random & 52.05 & 0.027 & 1.115 & $0.803-1.548$ & 0.649 & 0.517 & 1.000 & 0.956 \\
\hline \multicolumn{11}{|l|}{ Ethnicity } \\
\hline \multirow[t]{5}{*}{ Caucasian } & Cvs. G & Fixed & 25.45 & 0.235 & 1.084 & $0.961-1.222$ & 1.319 & 0.187 & 0.548 & 0.862 \\
\hline & CC vs. GG & Fixed & 21.99 & 0.262 & 1.152 & $0.901-1.472$ & 1.131 & 0.258 & 0.548 & 0.816 \\
\hline & CG vs. GG & Fixed & 20.91 & 0.270 & 1.137 & $0.941-1.373$ & 1.328 & 0.184 & 0.548 & 0.688 \\
\hline & $\mathrm{CC}+\mathrm{CG}$ vs. GG & Fixed & 0.00 & 0.466 & 1.182 & $0.988-1.413$ & 1.832 & 0.067 & 1.000 & 0.833 \\
\hline & CC vs. $C G+G G$ & Fixed & 45.88 & 0.086 & 1.040 & $0.837-1.291$ & 0.352 & 0.881 & 0.763 & 0.859 \\
\hline \multirow[t]{5}{*}{ Asian } & Cvs. G & Random & 94.58 & $\leq 0.001$ & 2.618 & $0.739-9.277$ & 1.491 & 0.136 & 0.734 & 0.797 \\
\hline & CC vs. GG & Fixed & 0.00 & 0.529 & 1.860 & $1.061-3.258$ & 2.168 & 0.030 & NA & NA \\
\hline & CG vs. GG & Random & 91.58 & $\leq 0.001$ & 1.752 & $0.471-6.517$ & 0.837 & 0.403 & 1.000 & 0.421 \\
\hline & $\mathrm{CC}+\mathrm{CG}$ vs. GG & Random & 91.78 & $\leq 0.001$ & 1.845 & $0.522-6.525$ & 0.951 & 0.342 & 1.000 & 0.468 \\
\hline & CC vs. $C G+G G$ & Fixed & 0.00 & 0.925 & 1.941 & $1.131-3.331$ & 2.407 & 0.016 & NA & NA \\
\hline \multicolumn{11}{|c|}{ Genotyping Method } \\
\hline TaqMan & C vs. G & Fixed & 0.00 & 0.604 & 1.148 & $0.968-1.362$ & 1.584 & 0.113 & 1.000 & 0.616 \\
\hline
\end{tabular}




\begin{tabular}{|c|c|c|c|c|c|c|c|c|c|c|}
\hline & CC vs. GG & Fixed & 0.00 & 0.677 & 1.304 & $0.931-1.828$ & 1.542 & 0.123 & 0.296 & 0.475 \\
\hline & CG vs. GG & Random & 70.75 & 0.033 & 1.159 & $0.692-1.940$ & 0.559 & 0.576 & 0.296 & 0.032 \\
\hline & $\mathrm{CC}+\mathrm{CG}$ vs. $\mathrm{GG}$ & Fixed & 47.78 & 0.147 & 1.169 & $0.908-1.505$ & 1.213 & 0.225 & 1.000 & 0.194 \\
\hline & CC vs. $C G+G G$ & Fixed & 13.27 & 0.316 & 1.207 & $0.896-1.624$ & 1.239 & 0.215 & 1.000 & 0.821 \\
\hline \multirow[t]{5}{*}{ PCR-RFLP } & C vs. G & Random & 82.35 & $\leq 0.001$ & 1.327 & $0.728-2.420$ & 0.925 & 0.355 & 0.452 & 0.517 \\
\hline & CC vs. GG & Random & 68.86 & 0.022 & 0.616 & $0.206-1.844$ & -0.866 & 0.386 & 0.734 & 0.572 \\
\hline & CG vs. GG & Random & 86.24 & $\leq 0.001$ & 1.374 & $0.566-3.337$ & 0.701 & 0.483 & 1.000 & 0.766 \\
\hline & $\mathrm{CC}+\mathrm{CG}$ vs. GG & Random & 87.81 & $\leq 0.001$ & 1.321 & $0.531-3.289$ & 0.598 & 0.550 & 1.000 & 0.817 \\
\hline & CC vs. $C G+G G$ & Random & 63.96 & 0.040 & 0.618 & $0.250-1.531$ & -1.039 & 0.299 & 1.000 & 0.657 \\
\hline \multicolumn{11}{|c|}{ Source of Controls } \\
\hline \multirow[t]{5}{*}{ HB } & C vs. G & Fixed & 32.59 & 0.217 & 0.919 & $0.750-1.125$ & -0.820 & 0.412 & 0.734 & 0.837 \\
\hline & CC vs. GG & Fixed & 52.39 & 0.098 & 1.075 & $0.687-1.682$ & 0.315 & 0.753 & 0.734 & 0.825 \\
\hline & CG vs. GG & Random & 73.58 & 0.010 & 0.585 & $0.317-1.080$ & -1.714 & 0.086 & 0.734 & 0.925 \\
\hline & $\mathrm{CC}+\mathrm{CG}$ vs. GG & Random & 80.19 & 0.002 & 0.620 & $0.318-1.208$ & -1.405 & 0.160 & 0.734 & 0.898 \\
\hline & $\mathrm{CC}$ vs. $\mathrm{CG}+\mathrm{GG}$ & Fixed & 46.16 & 0.134 & 1.211 & $0.804-1.826$ & 0.916 & 0.360 & 1.000 & 0.956 \\
\hline \multirow[t]{5}{*}{ PB } & C vs. G & Fixed & 41.99 & 0.142 & 1.079 & $0.940-1.239$ & 1.079 & 0.281 & 0.462 & 0.548 \\
\hline & CC vs. GG & Fixed & 53.38 & 0.092 & 1.140 & $0.857-1.517$ & 0.901 & 0.368 & 0.734 & 0.515 \\
\hline & CG vs. GG & Fixed & 39.23 & 0.160 & 1.189 & $0.955-1.479$ & 1.550 & 0.121 & 0.806 & 0.966 \\
\hline & $\mathrm{CC}+\mathrm{CG}$ vs. GG & Fixed & 10.90 & 0.344 & 1.255 & $0.995-1.508$ & 1.917 & 0.055 & 0.806 & 0.697 \\
\hline & CC vs. $C G+G G$ & Random & 68.17 & 0.024 & 0.919 & $0.571-1.481$ & -0.346 & 0.730 & 0.308 & 0.496 \\
\hline
\end{tabular}

GC: gastric cancer; PCR-RFLP: Polymerase Chain Reaction Restriction Fragment Length Polymorphism; HB: Hospital Based; PB: Population Based; NA: Not Applicable.

\section{HETEROGENEITY ANALYSIS AND SENSITIVITY ANALYSIS}

There was a significant heterogeneity under all five genetic models for both CRC and GC. As shown in tables 2 and 3, the $\mathrm{I}^{2}$ decreased obviously and $\mathrm{p}$-value exceeded 0.05 after excluding by source of controls for CRC and by ethnicity and source of controls for GC, indicating that ethnicity and source of controls are the major source of heterogeneity in this meta-analysis. Sensitivity analysis was performed by sequentially removing each study to examine the influence of the removed data to the overall ORs. No individ- ual study significantly altered the pooled ORs. Moreover, by limiting the meta-analysis to those studies in accordance with HWE, the sensitivity analysis was performed in another way. However, the corresponding ORs were not substantially altered in comparisons, indicating that our results were relatively robust.

\section{PUBLICATION BIAS}

Begg's test and Egger's test were calculated to assess the publication bias of literatures. The shapes of the funnel

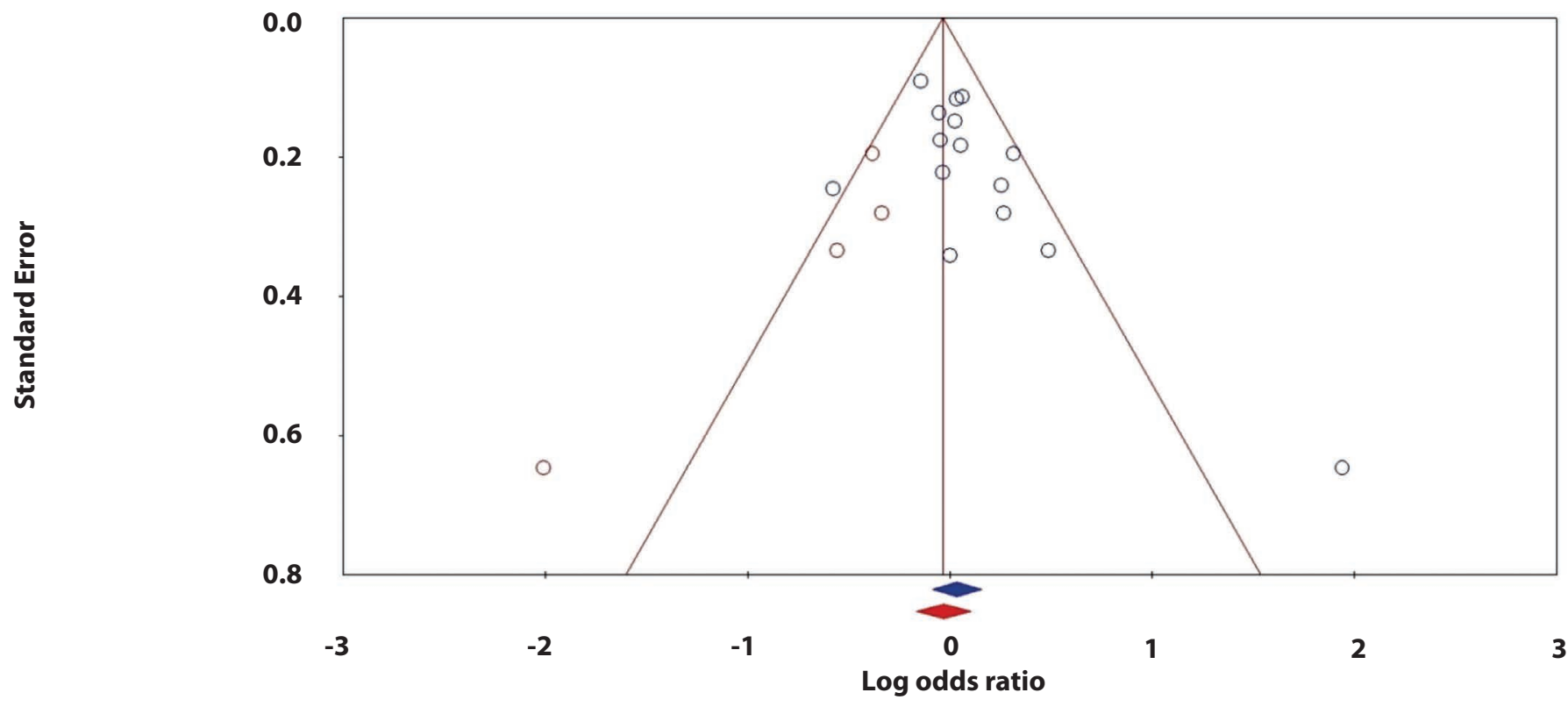

Fig. 3 Funnel plot for publication bias in the meta-analysis of IL-6 -174 G>C polymorphism with CRC under recessive model (CC vs. CG+GG). Blue line without and red line with trim and fill test. 
plot did not reveal any publication bias for IL- 6 polymorphism and GC risk under all five genetic models. In addition, No statistically significant difference was found in the Egger's test. However, there was a possible publication bias between IL- 6 polymorphism and CRC risk under recessive model $\left(\mathrm{P}_{\text {Beggs }}=0.113\right.$ and $\left.\mathrm{P}_{\text {Eggers }}=0.044\right)$. Therefore, we have used The Duval and Tweedie non-parametric "trim and fill" method to adjust publication bias. As shown in figure 3, meta-analysis with and without "trim and fill" did not draw different conclusion, suggesting that the results of synthetic analysis were robust.

\section{DISCUSSION}

To date, several case-control studies and meta-analyses have explored the association of IL-6 -174 G>C polymorphism on the susceptibility to CRC and GC. However, the small size, different genotyping methods and ethnicity, and the minor statistical power of the single epidemiological studies caused to the lack in consistency of those studies results. Thus, we did this meta-analysis to study the association of IL-6 -174 G>C polymorphism with susceptibility to CRC and GC. Our results suggested that the IL-6 $-174 \mathrm{G}>\mathrm{C}$ polymorphism was not significantly associated with increased risk of CRC and GC in overall population. In this meta-analysis, we found that similar mechanisms adapted by GC and CRC to development.

In the current meta-analysis based on 16 case-control available studies with 7,560 cases and 9,574 controls up to December 2018, our results indicated that there was no significant association between IL- $6-174 \mathrm{G}>\mathrm{C}$ polymorphism and CRC risk. In 2013, Hu et al. performed a meta-analysis of eleven individual studies with 6,481 cases and 7,935 controls to evaluate the association of IL-6 -174G>C polymorphism with risk of CRC. Similarly, they have not found a significant association between IL-6 $-174 G>C$ polymorphism and CRC (9). In 2016, Wang et al., conducted a meta-analysis to explore the association of polymorphisms at IL-6/JAK/STAT3 pathway genes with CRC risk. Their results indicated that IL-6 $-174 \mathrm{G}>\mathrm{C}$ polymorphism (allele model: $\mathrm{OR}=1.05,95 \% \mathrm{CI}=1.00,1.09$ ) and JAK2 (recessive model: $\mathrm{OR}=1.40,95 \% \mathrm{CI}=1.15,1.65$ ) were significantly associated with increased risk of CRC. However, their results showed that the IL- $6-174 \mathrm{G}>\mathrm{C}$ polymorphism was significantly associated with increased risk of CRC in Caucasians (40). Inconsistent with their results, our pooled data showed that the IL- $6-174 \mathrm{G}>\mathrm{C}$ polymorphism did not significantly associated with increased risk of CRC in Caucasians.

Previously, the relationship between IL-6 -174 G>C polymorphism and GC risk has been systematically evaluated, but their results had been conflicting and controversial. In the current meta-analysis we found that the IL- $6-174 \mathrm{G}>\mathrm{C}$ polymorphism was not significantly associated with increased risk of GC in overall. Recently, Wang et al., performed a meta-analysis to evaluate the association of IL-6 $-174 \mathrm{G}>\mathrm{C},-572 \mathrm{G}>\mathrm{C}$ and $-597 \mathrm{G}>\mathrm{A}$ with GC risk (8). Their results showed that IL- 6 polymorphisms were not associated with increased risk of GC. However, their results should be interpreted with caution due to the limited number of se- lected studies. Compared with their meta-analysis, we only focused on the association of IL- $6-174 \mathrm{G}>\mathrm{C}$ polymorphism with GC, while they analyzed different polymorphisms at other interleukin genes, including IL-6 rs1800796, IL-8 rs4073, IL-10 rs1800871, IL-10 rs1800872 and IL-10 rs1800896 polymorphisms with GC risk up to May 2018. Moreover, we perfumed subgroup analysis by genotyping methods and source of controls. In the same year, Liu et al., have performed a met-analysis of 13 studies (1,446 cases and 2,918 controls) to explore the roles of polymorphisms at IL-2, IL-4, IL-6 and IL-8 genes with GC risk (41). Their results revealed that IL- $6-572 \mathrm{C}>\mathrm{G}$ polymorphism was significantly associated with the risk of GC, but not IL-6 $-174 \mathrm{G}>\mathrm{C}$ polymorphism. Inconsistent with their results, we found a statistically significant association between IL-6 -174G>C polymorphism and GC risk in Asians under two genetic models i.e., homozygote (CC vs. GG: $\mathrm{OR}=1.860$, $95 \% \mathrm{CI} 1.061-3.258, \mathrm{p}=0.030$ ) and recessive (CC vs. CG+GG: $\mathrm{OR}=1.941,95 \%$ CI 1.131-3.331, $\mathrm{p}=0.016$ ).

Between-studies heterogeneity was demonstrated under all five genetic models for both CRC and GC, and we then conducted a subgroup analysis to explore the potential sources of heterogeneity, including ethnicity, source of controls, and genotyping methods. The results manifested that the heterogeneity could be mainly attributed by source of controls for CRC and by ethnicity and source of controls for GC. However, CRC and GC have a complex etiology and pathophysiology generated by the interaction of several genes and environmental factors $(7,42)$. Thus, besides the indeterminate number of characteristics that vary among studies, other confounding factors such as age, gender, lifestyle further contribute to between-study heterogeneity $(43,44)$. In addition, there was a publication bias across the selected studies in this meta-analysis. We suggested that the detected publication bias in a few studies could be attributed to relative small sample sizes in certain studies.

Though we included the latest data, several potential limitations must also be noticed in our meta-analysis. First, most of the selected studies were performed among Caucasians and Asians. Moreover, subgroup analysis based on ethnicity could not be assessed on CRC. Therefore, there was a lack of statistical power to better evaluate the association of IL-6 -174 G>C polymorphism with CRC and GC risk, especially in subgroup analysis. In addition, this bias may exist because the individuals may not be a full representative of the whole population. Thus, future studies with large sample sizes in different ethnicities are needed to determine the potential effects of ethnic variation on CRC and GC susceptibility. Second, our search results were restricted to publications in Chinese and English, other relevant published and unpublished studies, which are likely to have null results, were not included. Third, there was a significant publication bias in this meta-analysis under recessive model for CRC, which may be due to the small number of studies in the meta-analysis. Fourth, our meta- analysis was largely performed by unadjusted estimates, because of the limitations in selected studies that presented adjusted estimates. Finally, gene-gene and gene-environment interactions were not analyzed due to the lack of original data. It is possible that specific envi- 
ronmental and lifestyle factors may alter the association of IL-6 -174 G>C polymorphism with CRC and GC risk.

In conclusion, our meta-analysis suggested that the IL-6 -174 G>C polymorphism was not significantly associated with increased risk of CRC and GC in overall population. However, subgroup analysis by ethnicity showed that IL-6 -174 G>C polymorphism might be associated with an increased risk of GC in Asians. Due to the limitations, studies with larger sample size and in different ethnicities are needed to confirm our results.

\section{REFERENCES}

1. Namazi A, Abedinzadeh M, Nourbaksh P, Neamatzadeh H. Association between the XRCC3 Thr241Met polymorphism and risk of colorectal cancer: a meta analysis of 5,193 cases and 6,645 controls. Asian Pac J Cancer Prev 2015; 16(6): 2263-8.

2. Khoram-Abadi KM, Forat-Yazdi M, Kheirandish S, et al. DNMT3B -149 C\&gt;T and -579 G\&gt;T polymorphisms and risk of gastric and colorectal cancer: A meta-analysis. Asian Pacific J Cancer Prev 2016; 17(6): 3015-20.

3. Namazi A, Forat-Yazdi M, Jafari MA, et al. Association between polymorphisms of ERCC5 gene and susceptibility to gastric cancer: A systematic review and meta-analysis. Asian Pacific J Cancer Prev 2017; 18(10): 2611-7.

4. Arnold M, Sierra MS, Laversanne M, Soerjomataram I, Jemal A, Bray F. Global patterns and trends in colorectal cancer incidence and mortality. Gut 2017; 66(4): 683-91.

5. Khazaei S, Rezaeian S, Soheylizad M, Khazaei S, Biderafsh A. Global Incidence and Mortality Rates of Stomach Cancer and the Human Development Index: an Ecological Study. Asian Pac J Cancer Prev 2016; 17(4): 1701-4.

6. Forat-Yazdi M, Gholi-Nataj M, Neamatzadeh H, Nourbakhsh P, Shaker-Ardakani H. Association of XRCC1 Arg399Gln polymorphism with colorectal cancer risk: A huge meta analysis of 35 studies. Asian Pacific J Cancer Prev 2015; 16(8): 3285-91.

7. Namazi A, Forat-Yazdi M, Jafari M, et al. Association of interleukin-10-1082 A/G (rs1800896) polymorphism with susceptibility to gastric cancer: meta-analysis of 6,101 cases and 8,557 controls. Arq Gastroenterol 2018; 55(1): 33-40.

8. Wang X, Yang F, Xu G, Zhong S. The roles of IL- 6 , IL- 8 and IL-10 gene polymorphisms in gastric cancer: A meta-analysis. Cytokine 2018; 111: 230-6.

9. Hu JJ, Wang ZT, Zhong J. Lack of association between the interleukin 6 gene $-174 \mathrm{G} \& g t ; C$ polymorphism and colorectal cancer: evidence from a meta-analysis. Genet Mol Res 2013; 12(3): 2205-14.

10. Ma H, Sun G, Wang W, Zhou Y, Liu D, Tong Y, et al. Association Between Interleukin-6 -572 C>G and $-174 \mathrm{G}>\mathrm{C}$ Polymorphisms and Hypertension: A Meta-analysis of Case-control Studies. Medicine (Baltimore) 2016; 95(2): e2416.

11. Hefler LA, Grimm C, Lantzsch T, et al. Interleukin-1 and Interleukin-6 Gene Polymorphisms and the Risk of Breast Cancer in Caucasian Women. Clin Cancer Res 2005; 11(16): 5718-21.

12. Landi S, Moreno V, Gioia-Patricola L, et al. Association of common polymorphisms in inflammatory genes interleukin (IL) 6, IL8, tumor necrosis factor alpha, NFKB1, and peroxisome proliferator-activated receptor gamma with colorectal cancer. Cancer Res 2003; 63(13): 3560-6.

13. Theodoropoulos G, Papaconstantinou I, Felekouras E, et al. Relation between common polymorphisms in genes related to inflammatory response and colorectal cancer. World J Gastroenterol 2006; 12(31): 5037-43.

14. Gunter MJ, Canzian F, Landi S, Chanock SJ, Sinha R, Rothman N. Inflammation-Related Gene Polymorphisms and Colorectal Adenoma. Cancer Epidemiol Biomarkers Prev 2006; 15(6): 1126-31.

15. Gaustadnes M, Ørntoft TF, Jensen JL, Torring N. Validation of the use of DNA pools and primer extension in association studies of sporadic colorectal cancer for selection of candidate SNPs. Hum Mutat 2006; 27(2): 187-94.

16. Slattery ML, Wolff RK, Herrick JS, Caan BJ, Potter JD. IL6 genotypes and colon and rectal cancer. Cancer Causes Control 2007; 18(10): 1095-105.

17. Vogel U, Christensen J, Dybdahl M, et al. Prospective study of interaction between alcohol, NSAID use and polymorphisms in genes involved in the inflammatory response in relation to risk of colorectal cancer. Mutat Res 2007; 624(1-2): 88-100.
18. Wilkening S, Tavelin B, Canzian F, et al. Interleukin promoter polymorphisms and prognosis in colorectal cancer. Carcinogenesis 2008; 29(6): 1202-6.

19. Küry S, Buecher B, Robiou-du-Pont S, et al. Low-penetrance alleles predisposing to sporadic colorectal cancers: a French case-controlled genetic association study. BMC Cancer 2008; 8(1): 326.

20. Slattery ML, Wolff RK, Curtin K, et al. Colon tumor mutations and epigenetic changes associated with genetic polymorphism: Insight into disease pathways. Mutat Res Mol Mech Mutagen 2009; 660(1-2): 12-21.

21. Tsilidis KK, Helzlsouer KJ, Smith MW, et al. Association of common polymorphisms in IL10, and in other genes related to inflammatory response and obesity with colorectal cancer. Cancer Causes Control 2009; 20(9): 1739-51.

22. Vasku JA, Vasku A, Goldbergova M, Vasku V. Heterozygote AG variant of $-596 \mathrm{~A} / \mathrm{G}$ IL- 6 gene polymorphism is a marker for cutaneous T-cell lymphoma (CTCL). Clin Immunol 2004; 113(3): 256-60.

23. Ognjanovic S, Yamamoto J, Saltzman B, et al. Serum CRP and IL-6, genetic variants and risk of colorectal adenoma in a multiethnic population. Cancer Causes Control 2010; 21(7): 1131-8.

24. Čačev T, Jokić M, Lončar B, Križanac Š, Kapitanović S. Interleukin-6-174 G/C Polymorphism is Not Associated with IL-6 Expression and Susceptibility to Sporadic Colon Cancer. DNA Cell Biol 2010; 29(4): 177-82.

25. Abulí A, Fernández-Rozadilla C, Alonso-Espinaco V, et al. Case-control study for colorectal cancer genetic susceptibility in EPICOLON: previously identified variants and mucins. BMC Cancer $2011 ; 11(1)$ : 339.

26. Basavaraju U, Shebl FM, Palmer AJ, et al. Cytokine gene polymorphisms, cytokine levels and the risk of colorectal neoplasia in a screened population of Northeast Scotland. Eur J Cancer Prev 2015; 24(4): 296-304.

27. Banday MZ, Balkhi HM, Sameer AS, Chowdri NA, Haq E. Strong association of interleukin-6 -174G/C promoter single nucleotide polymorphism with a decreased risk of colorectal cancer in ethnic Kashmiri population: A case control study. Tumour Biol 2017; 39(3): 1010428317695940.

28. El-Omar EM, Rabkin CS, Gammon MD, et al. Increased risk of noncardia gastric cancer associated with proinflammatory cytokine gene polymorphisms. Gastroenterology 2003; 124(5): 1193-201.

29. Hwang I-R, Hsu P-I, Peterson LE, et al. Interleukin-6 genetic polymorphisms are not related to Helicobacter pylori-associated gastroduodenal diseases. Helicobacter 2003; 8(2): 142-8.

30. Kamangar F, Abnet CC, Hutchinson AA, et al. Polymorphisms in inflammation-related genes and risk of gastric cancer (Finland). Cancer Causes Control 2006; 17(1): 117-25.

31. Xing P, Xiao D, Zeng Q, Gao W. Relationship between cytokine gene polymorphisms on development and clinical characteristics of gastric adenocarcinoma. Chinese J Gen Surg 2006; 15: 659-63.

32. Deans C, Rose-Zerilli M, Wigmore S, et al. Host Cytokine Genotype is Related to Adverse Prognosis and Systemic Inflammation in Gastro-Oesophageal Cancer. Ann Surg Oncol 2007; 14(2): 329-39.

33. Gatti LL, Burbano RR, Zambaldi-Tunes M, et al. Interleukin-6 polymorphisms, Helicobacter pylori infection in adult Brazilian patients with chronic gastritis and gastric adenocarcinoma. Arch Med Res 2007; 38(5): 551-5.

34. Crusius JBA, Canzian F, Capella G, et al. Cytokine gene polymorphisms and the risk of adenocarcinoma of the stomach in the European prospective investigation into cancer and nutrition (EPICEURGAST). Ann Oncol 2008; 19(11): 1894-902.

35. Zhao Y, Xiao Y, Gao F. Relativity Research on the association between the interleukin- 6 gene polymorphisms and risk of gastric cancer in Wuwei Area of Gansu province. Clin J Med 2010; 38(1): 25-7.

36. Pohjanen V-M, Koivurova O-P, Mäkinen JM, et al. Interleukin 6 gene polymorphism -174 is associated with the diffuse type gastric carcinoma. Genes, Chromosom Cancer 2013; 52(10): 976-82.

37. Cao C. Multivariate analysis of the correlation between IL-6 174G/C gene polymorphism and gastric cancer. Mod Prev Med 2014; 41(20): 3756-8.

38. Sampaio AM, Balseiro SC, Silva MR, et al. Association Between IL-4 and IL-6 Expression Variants and Gastric Cancer Among Portuguese Population. GE Port J Gastroenterol 2015; 22(4): 143-52.

39. Attar M, Mansoori M, Shahbazi M. Interleukin-6 Genetic Variation and Susceptibility to Gastric Cancer in an Iranian Population. Asian Pac J Cancer Prev 2017; 18(11): 3025-9.

40. Wang S, Zhang W. Genetic variants in IL-6/JAK/STAT3 pathway and the risk of CRC. Tumor Biol 2016; 37(5): 6561-9.

41. Liu Y, Xu Y, Wang Y, Yao Y, Yang J. Associations between interleukin gene polymorphisms and the risk of gastric cancer: A meta-analysis. Clin Exp Pharmacol Physiol 2018; 45(12): 1236-44. 
42. Zare M, Jafari-Nedooshan J, Jafari M, et al. Relevance of hMLH1 -93G\&gt;A, 655A\&gt; G and 1151T\&gt;A polymorphisms with colorectal cancer susceptibility: a meta-analysis based on 38 case-control studies. Rev Assoc Med Bras 2018; 64(10): 942-51.

43. Moghimi M, Kargar S, Jafari MA, et al. Angiotensin Converting Enzyme Insertion/Deletion Polymorphism is Associated with Breast
Cancer Risk: A Meta-Analysis. Asian Pacific J Cancer Prev 2018; 19(11): 3225-31.

44. Abedinzadeh M, Zare-Shehneh M, Neamatzadeh $H$, Abedinzadeh M, Karami H. Association between MTHFR C677T polymorphism and risk of prostate cancer: Evidence from 22 studies with 10,832 cases and 11,993 controls. Asian Pacific J Cancer Prev 2015; 16(11): 4525-30. 